University of South Carolina

Scholar Commons

2-11-2002

\title{
Two Mechanisms of Blueshift of Edge Emission in InGaN-Based Epilayers and Multiple Quantum Wells
}

\author{
E. Kuokstis
}

J. W. Yang

Grigory Simin

University of South Carolina - Columbia, simin@engr.sc.edu

M. Asif Khan

R. Gaska

See next page for additional authors

Follow this and additional works at: https://scholarcommons.sc.edu/elct_facpub

Part of the Electromagnetics and Photonics Commons, and the Other Electrical and Computer Engineering Commons

\section{Publication Info}

Published in Applied Physics Letters, Volume 80, Issue 6, 2002, pages 977-979.

(C)Applied Physics Letters 2002, American Institute of Physics (AIP).

Kuokstis, E., Yang, J. W., Simin, G., Khan, M. A., Gaska, R., \& Shur, M. S. (11 February 2002). Two

Mechanisms of Blueshift of Edge Emission in InGaN-Based Epilayers and Multiple Quantum Wells.

Applied Physics Letters, 80 (6), 977-979. http://dx.doi.org/10.1063/1.1433164 


\section{Author(s)}

E. Kuokstis, J. W. Yang, Grigory Simin, M. Asif Khan, R. Gaska, and M. S. Shur 


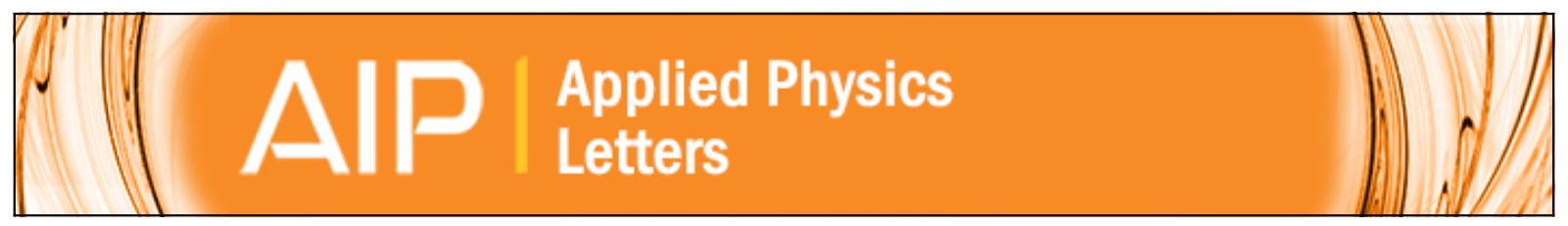

Two mechanisms of blueshift of edge emission in InGaN-based epilayers and multiple quantum wells

E. Kuokstis, J. W. Yang, G. Simin, M. Asif Khan, R. Gaska, and M. S. Shur

Citation: Applied Physics Letters 80, 977 (2002); doi: 10.1063/1.1433164

View online: http://dx.doi.org/10.1063/1.1433164

View Table of Contents: http://scitation.aip.org/content/aip/journal/apl/80/6?ver=pdfcov

Published by the AIP Publishing

\section{Articles you may be interested in}

Green light emission by InGaN/GaN multiple-quantum-well microdisks

Appl. Phys. Lett. 104, 102105 (2014); 10.1063/1.4868417

Two-color InGaN/GaN microfacet multiple-quantum well structures grown on Si substrate

J. Appl. Phys. 110, 083518 (2011); 10.1063/1.3653831

Effect of growth interruptions on the light emission and indium clustering of InGaN/GaN multiple quantum wells Appl. Phys. Lett. 79, 2594 (2001); 10.1063/1.1410362

Emission mechanisms of bulk GaN and InGaN quantum wells prepared by lateral epitaxial overgrowth Appl. Phys. Lett. 74, 1460 (1999); 10.1063/1.123581

"Blue" temperature-induced shift and band-tail emission in InGaN-based light sources

Appl. Phys. Lett. 71, 569 (1997); 10.1063/1.119797

\section{High-Voltage Amplifiers}

- Voltage Range from $\pm 50 \mathrm{~V}$ to $\pm 60 \mathrm{kV}$

- Current to 25A

\section{Electrostatic Voltmeters}

- Contacting \& Non-contacting

- Sensitive to $1 \mathrm{mV}$

- Measure to $20 \mathrm{kV}$
ENABLING RESEARCH AND

INNOVATION IN DIELECTRICS,

ELECTROSTATICS, MATERIALS, PLASMAS AND PIEZOS

TRek www.trekinc.com 


\title{
Two mechanisms of blueshift of edge emission in InGaN-based epilayers and multiple quantum wells
}

\author{
E. Kuokstis, ${ }^{\text {a) }}$ J. W. Yang, G. Simin, and M. Asif Khan ${ }^{\text {b) }}$ \\ Department of Electrical Engineering, University of South Carolina, Columbia, South Carolina 29208 \\ R. Gaska \\ Sensor Electronic Technology, Inc., 21 Cavalier Way, Latham, New York 12110 \\ M. S. Shur \\ ECSE, Rensselaer Polytechnic Institute, Troy, New York 12180 and Sensor Electronic Technology, Inc., \\ 21 Cavalier Way, Latham, New York 12110
}

(Received 13 July 2001; accepted for publication 3 November 2001)

\begin{abstract}
We present the results of a comparative photoluminescence (PL) study of GaN and InGaN-based epilayers, and InGaN/GaN multiple quantum wells (MQWs). Room-temperature PL spectra were measured for a very broad range of optical excitation from $10 \mathrm{~mW} / \mathrm{cm}^{2}$ up to $1 \mathrm{MW} / \mathrm{cm}^{2}$. In contrast to GaN epilayers, all In-containing samples exhibited an excitation-induced blueshift of the peak emission. In addition, the blueshift of the emission in the InGaN epilayers with the same composition as the quantum well was significantly smaller. The comparison of the blueshift in the "bulk" InGaN and in the MQWs allowed us to separate two different mechanisms responsible for this effect: (i) filling of the localized states in In-rich areas and (ii) screening of the polarization electric field in strained MQW structures. (C) 2002 American Institute of Physics.
\end{abstract}

[DOI: $10.1063 / 1.1433164]$

Indium-based III-nitride semiconductors are of great interest for applications in nearly all commercial lightemission systems since they allow band-gap tunability from the UV to the visible region of the spectrum. Ternary InGaNbased single or multiple quantum wells (MQWs) are the key structures for high-efficiency, long-lifetime violet, blue, and green light-emitting diodes and laser diodes. ${ }^{1-3}$ In spite of this importance, the edge emission mechanism for these InGaN layers and quantum wells is not yet fully understood. They exhibit a remarkably strong blueshift both with an increase in temperature and in carrier injection. ${ }^{4-6}$ In order to explain this blueshift, the idea of band-tail states was proposed with either a Gaussian-like density distribution ${ }^{7}$ or localization ${ }^{8}$ at quantum dots ${ }^{9}$ or disks. ${ }^{10}$ Attempts were also made to explain this edge emission peculiarity solely by the presence of a strain-induced piezoelectric field ${ }^{11}$ or by a combination of the field and the localized states. ${ }^{10}$

In this letter, we investigate the photoluminescence (PL) of high-quality InGaN-based epilayers and MQWs in a wide dynamic range of excitations. By comparing the PL from InGaN MQWs and thick InGaN epilayers of exactly the same composition as the well material, we were able to separate an excitation-induced blueshift from the band filling of the localized states from the screening of the internal electric field by the nonequilibrium photoexcited carriers. The value of the measured spectral shifts thus enabled us to calculate both the internal electric-field strengths in InGaN MQWs and the carrier density screening of the potential (using a triangular well model).

The samples for this study consisted of either single lay-

${ }^{a)}$ On leave from Department of Semiconductor Physics, Vilnius University, Sauletekio al. 9, Vilnius, 2040, Lithuania.

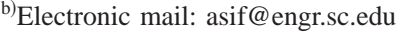

ers of $\operatorname{In}_{x} \mathrm{Ga}_{1-x} \mathrm{~N}$ or $\mathrm{In}_{x} \mathrm{Ga}_{1-x} \mathrm{~N} / \mathrm{GaN}$ MQWs. They were deposited on a $1-\mu \mathrm{m}$-thick GaN layer on a basal plane (0001) sapphire substrate using metalorganic chemical-vapor deposition. The $1-\mu \mathrm{m}$-thick $\mathrm{GaN}$ epilayer was grown at $980^{\circ} \mathrm{C}$ under a pressure of 76 Torr. More detailed information on the growth conditions may be found elsewhere. ${ }^{12}$ The InGaN/GaN MQW sample consisted of six periods of 4-nmthick quantum wells $\left(\operatorname{In}_{0.15} \mathrm{Ga}_{0.85} \mathrm{~N}\right)$ and 6-nm-thick $\mathrm{GaN}$ barriers, which were deposited at $731^{\circ} \mathrm{C}$ and capped by a 40-nm-thick GaN layer. The PL was excited using a $\mathrm{cw}$ He-Cd laser $(\lambda=325 \mathrm{~nm}$, maximum power $\sim 40 \mathrm{~mW})$ or by a pulsed $\mathrm{N}_{2}$ laser $(\lambda=337 \mathrm{~nm}$, pulse duration of $0.6 \mathrm{~ns}$, energy per pulse $\leqslant 5 \mu \mathrm{J}$ ). The laser beam was focused to a spot of $\sim 0.1 \mathrm{~mm}^{2}$. We were thus able to reach excitation power densities of $\sim 10^{-3}-40 \mathrm{~W} / \mathrm{cm}^{2}$ for the $\mathrm{cw}$ laser pump, and up to $\sim 10 \mathrm{MW} / \mathrm{cm}^{2}$ for the pulsed $\mathrm{N}_{2}$ laser excitation. The emitted light was collected perpendicular to the sample surface in a backscattering direction. The luminescence signal was analyzed by a SPEX550 monochromator with an UVenhanced liquid-nitrogen-cooled charge-coupled-device array. All the PL measurements were performed at room temperature.

At low excitation, PL spectra shapes are not excitation sensitive. Figure 1 shows typical PL spectra for the GaN epilayer (a) and InGaN MQWs (b) under different excitation power densities. At first, the increase of excitation power density does not change either peak position, or shape of the PL spectra for all the samples. In $\mathrm{GaN}$ under up to 50 $\mathrm{kW} / \mathrm{cm}^{2}$ excitation the spectrum is exciton originated: ${ }^{10}$ the predominant line corresponds to free excitons, whereas the low-energy wing structure of the spectrum could be explained by the LO phonon replicas. The InGaN samples (both epilayers and MQWs) demonstrate similar stability of 

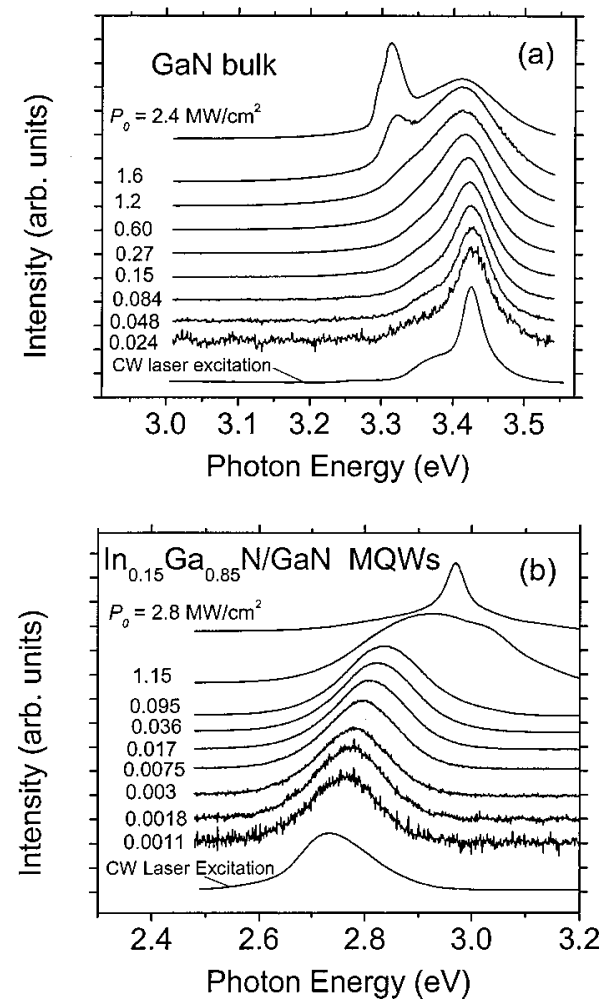

FIG. 1. Photoluminescence spectra of $\mathrm{GaN}$ epilayers (a) and $\mathrm{In}_{0.15} \mathrm{Ga}_{0.85} \mathrm{~N} / \mathrm{GaN}$ MQWs (b) under different $\mathrm{N}_{2}$ laser excitation power densities. The lowest curves correspond to $\mathrm{He}-\mathrm{Cd} \mathrm{cw}$ laser excitation with power density $<10 \mathrm{~W} / \mathrm{cm}^{2}$.

the PL spectra with excitation power, however, the full width at half maximum of the PL line is sufficiently broader $(\sim 180$ $\mathrm{meV}$ ) and the peak position remains unchanged unless the excitation power density exceeds $20 \mathrm{~W} / \mathrm{cm}^{2}$ for the InGaN MQWs, and $3 \mathrm{~kW} / \mathrm{cm}^{2}$ for the InGaN epilayer (see Fig. 2).

At higher excitation the PL properties of the GaN and InGaN samples are quite different. When the excitation power increases, the emission peak in $\mathrm{GaN}$ shifts towards lower energies (the maximum shift is $\sim 15 \mathrm{meV}$ ), the line broadens, and the structure of the LO phonons is no longer resolved. This PL spectra behavior for $\mathrm{GaN}$ epilayers under

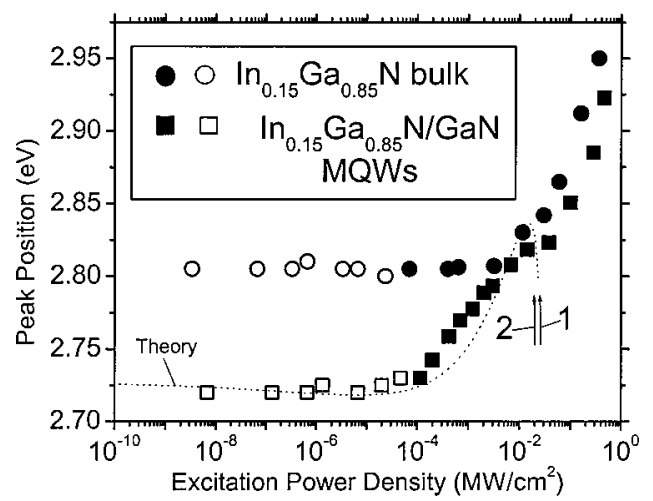

FIG. 2. Photoluminescence peak position dependences on excitation power density in $\mathrm{In}_{0.15} \mathrm{Ga}_{0.85} \mathrm{~N}$ epilayers (the upper dependence) and in $\mathrm{In}_{0.15} \mathrm{Ga}_{0.85} \mathrm{~N} / \mathrm{GaN}$ MQWs (the lower one). The open symbols correspond to $\mathrm{He}-\mathrm{Cd} \mathrm{cw}$ excitation, and the filled ones correspond to $\mathrm{N}_{2}$ laser pulsed excitation. Arrow 1 marks the excitation power density of complete screening of the electric field, and arrow 2 shows the validity limit of the model. The dotted line demonstrates the theoretical dependence of the emission maximum on excitation. strong excitation may be explained by a dense electron-hole plasma model, which has been successfully used for a number of highly pumped direct-gap semiconductors. ${ }^{13,14}$

In contrast to $\mathrm{GaN}$, the PL position both in the InGaN epilayers and InGaN MQWs shifts towards the high-energy side. However, the features of the excitation-induced blueshift in the bulk InGaN and in the MQW samples are different. As shown in Fig. 2, the total blueshift of the PL peak in bulk InGaN is about $150 \mathrm{meV}$, whereas it is more than 200 $\mathrm{meV}$ for the MQWs. For the MQW sample the blueshift occurs at the excitation power density, which is nearly two orders lower in comparison with the bulk InGaN. The blueshift at very high excitation in both cases demonstrates a remarkably similar behavior (see Fig. 2, experimental points at excitation $>\sim 100 \mathrm{~kW} / \mathrm{cm}^{2}$ ).

When the threshold of $1.0-1.4 \mathrm{MW} / \mathrm{cm}^{2}$ is exceeded the stimulated emission line appears, however, for $\mathrm{GaN}$ it is located on the long-wavelength side of the spontaneous PL spectrum, while for all InGaN samples it is located on the short-wavelength side [Figs. 1(a) and 1(b), upper curves]. Stimulated emission in all the samples can be explained by band-to-band recombination, ${ }^{13}$ whereas spontaneous PL in In-containing samples may be attributed to transitions between the density-of-state tales. ${ }^{7-10}$

We now analyze the spontaneous PL in the InGaN samples in more detail. We explain the PL behavior differences of the bulk $\operatorname{In}_{x} \mathrm{Ga}_{1-x} \mathrm{~N}$ and $\mathrm{In}_{x} \mathrm{Ga}_{1-x} \mathrm{~N} / \mathrm{GaN}$ MQWs by quantum confinement and the predominant strong piezoelectric field in thin quantum wells. Indeed, in hexagonalnitride MQWs, the quantum-confined Stark effect arises due to the piezoelectric field as well as due to spontaneous polarization. ${ }^{15}$ We analyzed the influence of nonequilibrium carriers on the position of PL spectra in the InGaN/GaN MQWs using a triangular well model. The photoexcited carriers screen the internal field. For an idealized case, neglecting thermal distribution in the bands, the emitted quantum energy $h \nu$ for band-to-band recombination in a quantum well in the presence of nonequilibrium electron-hole pair density $n$ can be expressed as

$$
h \nu=E_{g}(n)-e d F(n)+E_{e}(n)+E_{h}(n) .
$$

Here, $\operatorname{Eg}(n)$ is the carrier-density-dependent forbidden gap, which, taking into account band-gap renormalization, can be expressed as ${ }^{14} \quad E_{g}(n)=E_{g}(0)-\beta n^{1 / 3} \quad$ with $\beta=2$ $\times 10^{-8} \mathrm{eV} \mathrm{cm} ; d$ is the well width; $F(n)$ is the internal electric field strength, which can be expressed as $F(n) \approx F_{0}$ $-n e d / \epsilon \epsilon_{0}$ with maximum field strength $F_{0}$ (in the unexcited sample at limit $n \rightarrow 0$ ) and static relative dielectric constant $\epsilon$, which has been taken as ${ }^{5,16} 10$; and $E_{e, h}$ is the difference of the lowest-energy level from the triangular well bottom for electrons and holes, respectively, and can be calculated from $^{17}$

$$
E_{e, h} \cong\left(\frac{\hbar^{2}}{2 m_{e, h}}\right)^{1 / 3}\left[\frac{9 \pi e F(n)}{8}\right]^{2 / 3} .
$$

The effective masses of electrons or holes, $m_{e, h}$, used in the calculations have been assumed to be $0.25 m_{0}, m_{h} \cong m_{0}$, respectively. ${ }^{5,16}$ In order to compare our experimental results with the calculations, we expressed $h \nu$ as a function of excitation power density $P$ (in $\mathrm{MW} / \mathrm{cm}^{2}$ ), which for the case of 
predominantly square-law recombination are related as $n$ $=\sqrt{P \alpha / h \nu \gamma}$, where $\alpha$ is the absorption coefficient for laser light and has been taken as ${ }^{18} 1.5 \times 10^{5} \mathrm{~cm}^{-1}$, and $\gamma$ is the square-law recombination coefficient and has been taken as ${ }^{19}$ $4.8 \times 10^{-11} \mathrm{~cm}^{3} \mathrm{~s}^{-1}$.

The results of these calculations for InGaN/GaN MQWs are illustrated in Fig. 2 by the dotted line. This rather crude model gives remarkably good agreement between experimental data and theoretical estimations for more than six orders of excitation power density (up to $\sim 10 \mathrm{~kW} / \mathrm{cm}^{2}$ ). Note that a number of other effects were not included in our model (we neglected the two-dimensional nature of the system, carrier distribution in the barriers and the wells, excitonic and nonradiative recombination channels, possible recombination coefficient change due to separation of the carriers in wells, etc.). These effects can mainly change the concentration of electron-hole pairs corresponding to a given excitation power density, which results in just the "horizontal" shift of the theoretical curve in Fig. 2. However, this shift is quite small due to a logarithmic scale of the power density axis. In these calculations we have adjusted just two parameters: the maximum electric-field strength $F_{0}$ and the initial PL peak position. The value of $F_{0}$ was found to be equal to $1.87 \times 10^{6} \mathrm{~V} / \mathrm{cm}$, which is quite similar to other evaluations in similar semiconductor structures. $5,10,20$ The estimation of the built-in (mainly piezoelectric) field in $\mathrm{In}_{0.15} \mathrm{Ga}_{0.85} \mathrm{~N} / \mathrm{GaN}$ MQWs following Refs. 15 and 21 yields the value of $1.73 \mathrm{MV} / \mathrm{cm}$, which is close to that obtained experimentally. Complete screening of the field is achieved at carrier densities $\sim 10^{20} \mathrm{~cm}^{-3}$ (arrow 1 in Fig. 2). Note that the infinite triangular quantum well model is valid only up to a carrier density $\sim 8 \times 10^{19} \mathrm{~cm}^{-3}$ (arrow 2 in Fig. 2). As can be seen from Fig. 2, the region of excitation power density around $\sim 10 \mathrm{~kW} / \mathrm{cm}^{2}$ is the starting point for the blueshift of the PL spectrum of bulk InGaN material, as well as some "slowing" of the blueshift in MQWs. Beyond this pumping level, a very similar PL maximum shift is observed for both the bulk and the MQW samples. This blueshift in Incontaining III-N samples may be attributed to band-tail filling by carriers. ${ }^{7-10}$ Recently, we have also observed the clearly resolved blueshift maximum [as a result of the field screening leading to the competition between the second and the last two terms in formula (1)] in quaternary AlInGaN MQWs with a very low fraction of In, and which thus have no localized In-related potentials and tails. ${ }^{22}$

In summary, we have shown that an excitation-induced blueshift of PL spectra is observed in bulk InGaN epilayers, as well as in InGaN/GaN MQWs. The comparison of PL data in $\mathrm{GaN}$, InGaN, and InGaN/GaN MQW samples, as well as the theoretical estimations using the triangular potential-well model have shown that the blueshift observed in bulk mate- rials is caused by filling of band-tail states, which result from potential fluctuations due to an inhomogeneous distribution of In-rich dots. In InGaN/GaN MQWs, with increased excitation levels, the blueshift at first is from screening of the internal electric field (by photoinduced carriers) and later by filling of band-tail states.

This work at USC was supported by the Ballistic Missile Defense Organization (BMDO) under Army SMDC Contract No. DASG60-98-1-0004, monitored by Terry Bauer, Dr. Brian Strickland, and Dr. Kepi Wu. The work at SET, Inc. was supported by the Office of Naval Research under Small Business Technology Transfer Phase II Contract No. N00014-01-C-0195 and monitored by Dr. Y.-S. Park.

${ }^{1}$ S. Nakamura, M. Senoch, S. Nagahama, N. Iwasa, T. Yamada, T. Matsushita, H. Kiyoku, and Y. Sugimoto, Jpn. J. Appl. Phys., Part 2 35, L74 (1996).

${ }^{2}$ S. Nakamura and G. Fasol, The Blue Laser Diode (Springer, Heidelberg, 1997).

${ }^{3}$ S. J. Pearton, J. C. Zolper, R. J. Shul, and F. Ren, J. Appl. Phys. 86, 1 (1999).

${ }^{4}$ P. Perlin, M. Osiński, and G. Eliseev, Mater. Res. Soc. Symp. Proc. 449, 1173 (1996).

${ }^{5}$ T. Takeuchi, S. Sota, M. Katsuragawa, M. Komori, H. Takeuchi, H. Amano, and I. Akasaki, Jpn. J. Appl. Phys., Part 2 36, L382 (1997).

${ }^{6}$ Y. Narukawa, Y. Kawakami, S. Fujita, and S. Fujita, Phys. Rev. B 55, R1938 (1997).

${ }^{7}$ P. G. Eliseev, P. Perlin, J. Lee, and M. Osiński, Appl. Phys. Lett. 71, 569 (1997).

${ }^{8}$ S. Chichibu, T. Azuhata, T. Sota, and S. Nakamura, Appl. Phys. Lett. 70, 2822 (1997).

${ }^{9}$ K. P. O’Donnell, R. W. Martin, and P. G. Middleton, Phys. Rev. Lett. 82, 237 (1999).

${ }^{10}$ S. Chichibu, T. Sota, K. Wada, and S. Nakamura, J. Vac. Sci. Technol. B 16, 2204 (1998).

${ }^{11}$ P. Riblet, H. Hirayama, A. Kinoshita, A. Hirata, T. Sugano, and Y. Aoyagi, Appl. Phys. Lett. 75, 2241 (1999).

${ }^{12}$ M. A. Khan, S. Krishnankutty, R. A. Skogman, J. N. Kuznia, D. T. Olson, and T. George, Appl. Phys. Lett. 65, 520 (1994).

${ }^{13}$ V. G. Lysenko, V. I. Revenko, T. G. Tratas, and V. B. Timofeev, Sov. Phys. JETP 41, 163 (1975).

${ }^{14}$ W. F. Brinkman and T. M. Rice, Phys. Rev. B 7, 1508 (1973).

${ }^{15}$ F. Bernardini, V. Fiorentini, and D. Vanderbilt, Phys. Rev. B 56, R10024 (1997).

${ }^{16}$ D. W. Palmer, www.semiconductors.co.uk, 2001.04.

${ }^{17}$ C. Weisbuch and B. Vinter, Quantum Semiconductor Structures (Academic, London, 1991).

${ }^{18}$ H. Anger, D. Brunner, F. Freudenberg, O. Ambacher, M. Stutzmann, R. Höpler, T. Metzger, E. Born, G. Dollinger, A. Bergmaier, S. Karsch, and H.-J. Körner, Appl. Phys. Lett. 71, 1504 (1997).

${ }^{19}$ A. Dmitriev and A. Oruzheinikov, J. Appl. Phys. 86, 3241 (1999).

${ }^{20}$ S. F. Chichibu, T. Sota, K. Wada, O. Brandt, K. H. Ploog, S. P. DenBaars, and S. Nakamura, Phys. Status Solidi A 183, 91 (2001).

${ }^{21}$ R. Cingolani, A. Botcharev, H. Tang, H. Markoç, G. Traetta, G. Coli, M. Lomascolo, A. Di Carlo, F. Della Sala, and P. Lugli, Phys. Rev. B 61, 2711 (2000).

${ }^{22}$ E. Kuokstis, J. Zhang, J. W. Yang, G. Simin, M. Asif Khan, R. Gaska, and M. S. Shur, Phys. Status Solidi B 228, 559 (2001). 